\title{
SERUM LEPTIN AND ADIPOSITY INDICATORS IN INFERTILE FEMALES OF REPRODUCTIVE AGE GROUP
}

\author{
ARUL SENGHOR, MEERA SHIVASHEKAR*, VM VINODHINI
}

Department of Biochemistry, SRM Medical College Hospital \& Research Centre, Kattankulathur, Kanchipuram, Tamil Nadu, India. Email: mshivasekar@gmail.com

Received: 10 November 2017, Revised and Accepted: 21 March 2018

\section{ABSTRACT}

Objectives: This study was designed to evaluate the interrelationship of adiposity indicators such as body mass index (BMI), waist circumference (WC), hip circumference, waist-hip ratio and waist height ratio, body adiposity index (BAI) in obese and non-obese female individuals unable to conceive. And also to correlate serum leptin levels with adiposity indicators in infertile females of reproductive age group.

Methods: Infertile females of reproductive age group were classified as obese and non-obese individuals based on BMI and waist-hip ratio. Serum leptin levels were estimated and correlated with adiposity indicators using Pearson's correlation statistical analysis.

Results: The mean age and duration of infertility were high in obese than the non-obese infertile females. Leptin showed significant positive correlation with WC, waist hip ratio (WHR), and waist/height ratio than the routinely done BMI which had low positive correlation. BAI had mild positive correlation with leptin.

Conclusion: WC and WHR are simple convenient non-invasive tools that can differentiate the type of obesity. Adiposity indicators guide the physician to identify the infertile female individuals who require preliminary steps toward weight reduction before starting the right line of therapy to correct their hormonal imbalance.

Keywords: Infertility, Obesity, Leptin, Waist circumference, Waist to height ratio, Body adiposity index.

(c) 2018 The Authors. Published by Innovare Academic Sciences Pvt Ltd. This is an open access article under the CC BY license (http://creativecommons. org/licenses/by/4. 0/) DOI: http://dx.doi.org/10.22159/ajpcr.2018.v11i6.23569

\section{INTRODUCTION}

Infertility is growing at an alarming pace, which is a heartbreaking and painful condition for the couples. Globally, every year 60-80 million couples suffer from infertility as estimated of which India alone is probably between 15 and 20 million. The incidence of female infertility is at the rise and is currently $37 \%$ of all the infertile couples [1,2]. Obesity is an important and common health issue in women of reproductive age group. Although critical body mass of adipose tissue is essential for normal female reproductive function; excess adipose tissue has shown to result in derangement of reproductive hormones. The prevalence of overweight and obesity is common among adolescents in rural areas also; due to excess calorie intake and less calorie expenditure that leads to lifestyle-related disorders [3]. Obesity and overweight females definitely have negative impact on their reproductive health. Studies have demonstrated that obese women experience increased duration for spontaneous pregnancy and also pregnancy rates are decreased $[4,5]$.

Infertility occurs in obese women due to many reasons such as impaired ovarian follicular development, poor qualitative oocyte, defective fertilization, embryo development, and implantation. Anovulatory infertility is revealed as one of the most common causes in overweight and obese individuals. Studies have proved that anovulation is the effect of hyperinsulinemia, insulin resistance, and hyperandrogenism on the ovary [6].

Adipose tissue is considered as an endocrine organ that produces various signaling molecules called adipocytokines. Adipocyte-derived leptin is a peripheral indicator of the adequacy of nutritional status for reproductive functions. Leptin is also produced by human ovarian follicles. Leptin is important for normal menstrual cycle and ovulation. A study concluded that obesity is one of the main causes of infertility that may be controlled by regulating the leptin concentration in unexplained infertile females [7]. In a study, overweight and women who are obese were reported to have reduced fecundity even with regular menses [8]. Combination of various possible causes and increased circulating levels of leptin on the granulosa and theca cell gives rise to infertility [9]. Simple effective and available investigations act as a tool in devising the treatment strategies for the infertile patients. Taking into consideration the pathological link between adiposity and hormonal imbalance in obese infertile individuals, the study aims to analyze and compares the various adiposity indicators and correlate with serum leptin levels and to identify and focus on the useful indicators applicable as screening tool in infertile individuals.

\section{METHODS}

This cross-sectional observational study included 60 infertile out of 82 infertile participants in the age group of 20-40 years attending the infertility clinic of SRM Medical College Hospital and Research Centre. The data of each patient were recorded in a pre-designed pro forma after informed consent. A detailed history and preliminary clinical examination were carried.

\section{Ethical consideration}

All study protocols were reviewed and approved by the Institutional Ethical Committee (IEC: No: 963/IEC/2016). The purpose of the protocol was explained and written informed consent was obtained from the participants.

\section{Anthropometric indicators}

All the participant's height, weight, waist circumference (WC), and hip circumference (HC) were measured. Height was measured to the nearest meter; weight was measured to the nearest $0.1 \mathrm{~kg}$ on an electric scale. Body mass index (BMI) was measured with weight in $\mathrm{kg}$ and height in meter $\left(\mathrm{kg} / \mathrm{m}^{2}\right)$. WC is measured with an inch tape at the narrowest circumference of the natural waist. HC is measured around 
the widest portion of the buttocks. Waist-hip ratio is calculated by dividing WC with that of HC. It is an effective way to assess the regional fat distribution. Waist to height ratio (WC/Ht ratio) is calculated by dividing WC with that of height in same units. Body adiposity index (BAI) is calculated with a formula: [hip circumference $\div$ height1.5]. As per the classification of BAI for women based on the age 20-40 years: $\mathrm{BAI}<21 \%$ are underweight, $21-33 \%$ is healthy, $>33-38 \%$ is overweight, and $>39 \%$ is obese [10]

\section{Inclusion criteria}

Primary or secondary infertile females with the age group of 20-40 years with complaints of irregular menstrual cycles or ovulatory dysfunction (ultrasound finding) or polycystic ovary syndrome (According to Rotterdam's criteria) were recruited. They were divided as Group A infertile individuals $(\mathrm{n}=42)(\mathrm{BMI} \geq 23)$ and Group B lean infertile individuals $(\mathrm{n}=18)(\mathrm{BMI} \leq 22.9)$.

\section{Exclusion criteria}

The participants with infertile male partners, uterine anomaly, tubal lesions, pregnancy, diabetes mellitus, hypertension, and recent infections were excluded from the study.

\section{Sample collection and analysis}

Fasting venous samples were collected from the participants using standard venipuncture technique. Serum was separated and stored for further biochemical analysis. The samples were analyzed in batches in the laboratory according to standard operating procedures within 30 min of being thawed.

Serum leptin was assayed in 96 well ELISA human leptin kit (DBC) using a BIO-RAD ELISA reader. The limit of detection for leptin is $0.50 \mathrm{ng} / \mathrm{L}$.

\section{Statistical analysis}

Statistical analysis was performed using SPSS software, version 21. The results were expressed as mean \pm standard deviation (SD). $\mathrm{p}<0.05$ was considered as statistically significant. The correlation of leptin with adiposity indicators in obese infertile individuals were evaluated by Pearson correlation coefficient.

\section{RESULTS}

The 60 infertile female participants of reproductive age group were categorized based on WHR and WC. The mean age of the infertile individuals was $28 \pm 5$ years. Among the participants, $70 \%$ were overweight and $30 \%$ were lean infertile females.

Table 1 shows the data of Anthropometric indicators in obese and lean infertile individuals expressed in mean and SD. The mean age (31 \pm 5.0$)$ years and average duration of infertility $(8.2 \pm 3.48)$ years in obese is increased as compared to the lean infertile individuals. Waist and HC were increased in obese individuals and was found to be significant. Likewise, the $\mathrm{WC} / \mathrm{Ht}$ ratio and BAI showed statistically significant $\mathrm{p}$ value in obese individuals ( $\mathrm{p}$ of 0.001 and 0.014 ), respectively. As expected serum leptin levels concentration was increased in obese infertile females than the lean individuals, with statistically significant $\mathrm{p}<0.0001$

As shown in Table 2, positive correlation of leptin with age, duration of infertility and adiposity indicators in obese individuals were observed. The indicators that considered $\mathrm{HC}$ and height such as BAI $(\mathrm{r}=+0.35)$ and $\mathrm{WC} / \mathrm{Ht}$ ratio $(\mathrm{r}=+0.32)$ showed moderately positive correlation with leptin. Whereas, WC and WHR showed a moderately positive significant correlation, $(\mathrm{r}=+0.49$ and +0.45$)$, respectively, with significant increase in leptin levels.

\begin{tabular}{lll}
\hline Correlation & Negative & Positive \\
\hline Small & -0.3 to -0.1 & 0.1 to 0.3 \\
Medium & -0.5 to- 0.3 & 0.3 to 0.5 \\
Strong & -1.0 to -0.5 & 0.5 to 1.0 \\
\hline
\end{tabular}

\section{DISCUSSION}

The negative effects of obesity in reproductive life of women are a complex and multifactorial consequence. There are various mechanisms involved to explain the association between obesity and inability to conceive in spite of unprotected regular sexual intercourse. The present study has revealed that WC and WHR are important anthropometric measurements to evaluate visceral adiposity and hormonal pattern in obese females presenting with primary or secondary infertility.

The cross-sectional study has evaluated and compared the adiposity indices in infertile female participants. The age of the participants with obesity was in their early thirties and also had increased duration of infertility that indicates the shift in the potential of conception. Female age is an important criterion where the fertility rate decreases with the advancement of age, decides the quality of the oocyte, reduces the chance of conception and are associated with pregnancy complications [11]. BMI alone does not provide much information apart from the type of obesity. Thereby along with BMI; WC, WHR, WC/Ht ratio, and BAI provide information that characterizes women with visceral obesity and information related to the abnormal hormonal pattern in them.

In this study, the serum leptin levels are elevated in obese individuals as compared to their lean counterparts. This is supported by the research work that demonstrated the linear correlation of leptin with BMI [6]. Leptin is an adipocytokine produced by the visceral adipose tissue that influences gonadal function through modulating steroidogenesis in ovarian granulosa cells. Leptin has a critical role in the control of ovulation, development of a dominant follicle and oocyte maturation $[12,13]$.

In individuals with increased WC, the study demonstrated increased WHR, WC/Ht ratio, BAI, and leptin levels. Furthermore, the correlation

Table 1: Anthropometric and biochemical characteristics of obese and lean female infertile individuals

\begin{tabular}{llll}
\hline Characteristics & $\begin{array}{l}\text { Obese } \\
\text { infertile } \\
\text { females }\end{array}$ & $\begin{array}{l}\text { Lean } \\
\text { infertile } \\
\text { females }\end{array}$ & p value \\
\hline Age & $31 \pm 5.0$ & $26 \pm 3.55$ & $0.001^{* *}$ \\
Duration of infertility & $8.2 \pm 3.48$ & $5.32 \pm 2.74$ & $0.012^{* *}$ \\
Weight (kg) & $62.6 \pm 4.6$ & $54.9 \pm 1.6$ & $<0.05^{*}$ \\
Height (m) & $1.56 \pm 0.024$ & $1.59 \pm 0.026$ & $\mathrm{NS}$ \\
BMI & $24.88 \pm 1.78$ & $21.77 \pm 0.55$ & $<0.05^{*}$ \\
WC (cm) & $91.89 \pm 5.95$ & $82.72 \pm 1.19$ & $0.005^{* *}$ \\
HC (cm) & $112.03 \pm 2.86$ & $98.45 \pm 2.06$ & $0.002^{* *}$ \\
WHR & $0.89 \pm 0.03$ & $0.84 \pm 0.024$ & $0.02^{* *}$ \\
WC/Ht ratio & $0.61 \pm 0.04$ & $0.51 \pm 0.02$ & $0.001^{* *}$ \\
BAI & $38.11 \pm 1.95$ & $30.23 \pm 1.50$ & $0.014^{* *}$ \\
Leptin (ng/mL) & $59.58 \pm 17.65$ & $45.43 \pm 17.79$ & $0.0001^{* * *}$ \\
\hline
\end{tabular}

Values are expressed in mean \pm standard deviation. $\mathrm{P}<0.05$ is considered significant. NS: Not significant. ${ }^{* *}$ Significant, ${ }^{* * *}$ Highly significant. WC/Ht ratio Waist/height ratio, BMI: Body mass index, WC: Waist circumference, HC: Hip circumference, BAI: Body adiposity index, WHR: Waist hip ratio

Table 2: Correlation of leptin with age, duration of infertility, WC, WHR, BAI in obese and lean female infertile individuals

\begin{tabular}{ll}
\hline Infertile individuals & Obese individuals \\
\hline Age (years) & +0.35 \\
Duration of infertility (years) & +0.36 \\
BMI & +0.34 \\
Waist circumference & +0.49 \\
WHR & +0.45 \\
WC/Ht ratio & +0.32 \\
BAI & +0.35 \\
\hline
\end{tabular}

WC/Ht ratio: Waist/height ratio, BMI: Body mass index, WC: Waist circumference, BAI: Body adiposity index, WHR: Waist hip ratio 
of leptin with these adiposity indices [14] adds more impact on the fact that increase in visceral adipose tissue is associated with abnormal hormonal pattern which is influenced by the leptin levels that affects hypothalamic-pituitary-gonadal axis.

This is explained by the fact that increased visceral adiposity results in defective insulin post-receptor autophosphorylation which leads to insulin resistance. This, in turn, results in elevated insulin levels which lower sex hormone binding globulin with increased ovarian androgen production, elevated estradiol levels and altered luteinizing hormone/follicle-stimulating hormone which is inextricably linked to anovulation. The finding is consistent with the studies which proved that obesity-related insulin resistance plays a key role in ovulatory dysfunction $[15,16]$. Lifestyle modification and insulin sensitizers lead to weight reduction and favor ovulation in individuals with upper body obesity [17]. The significant positive correlation of leptin with WC and WHR justifies the need of weight reduction before the right line of treatment that includes the carrying out assisted reproductive technology which is expensive [18]. Although serum leptin levels were high normal in lean infertile individuals; further research work is necessary to reveal the underlying mechanism in lean infertile female individuals.

\section{CONCLUSION}

The study concludes that weight reduction is the only way to increase the chance of ovulation and conception in overweight and obese women and also help the physician to take the right holistic approach. Leptin, WC, and WHR are better predictor of adiposity and reliable measure than BMI alone.

\section{ACKNOWLEDGMENTS}

The authors would like to acknowledge the great advice and comments given by Professor Dr. S. Muthulakshmi from Infertility Clinic. This research did not receive any specific grant from funding agencies.

\section{CONFLICT OF INTEREST}

The authors declare that there is no conflict of interest.

\section{REFERENCES}

1. Adamson PC, Krupp K, Freeman AH, Klausner JD, Reingold AL, Madhivanan P. Prevalence and correlates of primary infertility among young women in Mysore, India. Indian J Med Res 2011;134:440-6.

2. Kumar D. Prevalence of Female Infertility and its Socio-economic Factors in Tribal Communities of Central India. Rural and Remote Health; 2007. Available from: http://www.rrh.org.au. [Last cited on 2013 Dec 27].
3. Mithra PP, Kumar P, Kamath VG, Kamath A, Unnikrishnan B, Rekha T, et al. Lifestyle factors and obesity among adolescents in rural South India. Asian J Pharm Clin Res 2015;8:81-3.

4. Law DC, Maclehose RF, Longnecker MP. Obesity and time of pregnancy. Hum Reprod 2007;22:414-20.

5. Wise LA, Rothman KJ, Mikkelsen EM, Sorensen HT, Riis A, Hatch EE. An internet based prospective study of body size and time-to-pregnancy. Hum Reprod 2010;25:253-64.

6. Jungheim ES, Travieso JL, Hopeman MM. Weighing the impact of obesity on female reproductive function and fertility. Nutr Rev 2013;71:3-8

7. Kumari P, Jaiswar SP, Shankhwar P, Deo S, Ahmed K, Iqbal S, et al. Leptin as a predictive marker in unexplained infertility in North Indian population. J Clin Diagn Res 2017;11:QC28-31.

8. Rittenberg V, Seshadri S, Sundara SK, Sobaleva S, Oteng-Ntim E, El-Toukhy T. Effect of body mass index in IVF treatment outcome: An updated systematic review and meta-analysis. Reprod Biomed Online 2011;23:421-39.

9. Dag ZO, Dilbaz B. Impact of obesity on infertility in women. J Turk Ger Gynecol Assoc 2015; 16:111-7.

10. Freedman DS, Thornton J, Pi-Sunyer FX, Heymsfield SB, Wang J, et al. The body adiposity index (hip circumference $\div$ height 1.5 ) is not a more accurate measure of adiposity than is BMI, waist circumference, or hip circumference. Obesity (Silver Spring) 2012;20:2438-44

11. Amato MC, Guarnotta V, Forti D, Donatelli M, Dolcimascolo S, Giordano C, et al. Metabolically healthy polycystic ovary syndrome (MH-PCOS) and metabolically unhealthy polycystic ovary syndrome (MU-PCOS): A comparative analysis of four simple methods useful for metabolic assessment. Hum Reprod 2013;28:1919-28.

12. Karoutsos P, Karoutsou E, Karoutsos D. Obesity and female fertility: The bridging role of leptin. J Data Mining Genomics Proteomics 2017;8:209.

13. Di Yorio MP, Bilbao MG, Biagini-Majorel AM, Faletti AG. Ovarian signalling pathways regulated by leptin during the ovulatory process. Reproduction 2013;146:647-58.

14. Ali SH, Al-Nuaimi AM, Al-Musawi BM. Serum irisin and leptin levels in obese and non-obese women with polycystic ovary syndrome with reference to glucose homeostasis. Int J Pharm Pharm Sci 2016;8:276-83.

15. Ebrahimi-Mamaghani M, Saghafi-Asl M, Pirouzpanah S, Aliasgharzadeh A, Aliashrafi S, Rezayi N, et al. Association of insulin resistance with lipid profile, metabolic syndrome, and hormonal aberrations in overweight or obese women with polycystic ovary syndrome. J Health Popul Nutr 2015;33:157-67.

16. Tsutsumi R, Webster NJ. GnRH pulsatility, the pituitary response and reproductive dysfunction. Endocr J 2009;56:729-37.

17. Nehra J, Kaushal J, Singhal SR, Ghalaut VS. Comparision of myoinositol versus metformin on anthropometric parameters in polycystic ovarian syndrome in women. Int J Pharm Pharm Sci 2017;9:144-8.

18. Desai A, Shirode AR, Bhanushali M, Kadam VJ. Assisted reproductive technology (ART): Combating infertility. Asian J Pharm Clin Res 2011;4:18-22 